\title{
A methodology to measure the interface shear strength by means of the fiber push-in test
}

\author{
M. Rodríguez a , J.M. Molina-Aldareguía a , C. González ${ }^{\mathrm{a}, \mathrm{b}}$, J. LLorca ${ }^{\mathrm{a}, \mathrm{b}, *}$
}

\begin{abstract}
A B S T R A C T
A methodology is presented to measure the fiber/matrix interface shear strength in composites. The strategy is based on performing a fiber push-in test at the central fiber of highly-packed fiber clusters with hexagonal symmetry which are often found in unidirectional composites with a high volume fraction of fibers. The mechanics of this test was analyzed in detail by means of three-dimensional finite element simulations. In particular, the influence of different parameters (interface shear strength, toughness and friction as well as fiber longitudinal elastic modulus and curing stresses) on the critical load at the onset of debonding was established. From the results of the numerical simulations, a simple relationship between the critical load and the interface shear strength is proposed. The methodology was validated in an unidirectional C/epoxy composite and the advantages and limitations of the proposed methodology are indicated.
\end{abstract}

\section{Introduction}

Composite materials contain a large volume fraction of small diameter fibers, which leads to a very large interface area per unit volume (of the order of $10^{5} \mathrm{~m}^{2} / \mathrm{m}^{3}$ in typical carbon fiber composites). Obviously, composite properties are controlled to large extent by the interface properties and, in particular, interface strength and toughness play a dominant role in the mechanical performance of fiber-reinforced composites. Despite the importance of this topic, there is no consensus about the most accurate technique to characterize the interface mechanical properties and different tests are used for this purpose [1]. They can be broadly divided into two groups depending on whether the measurements are carried out in microcomposites made up of single fibers or fiber tows embedded in the matrix or from the actual composite samples.

The measurement techniques applied to single-fiber microcomposites include the fiber fragmentation test [2], the single fiber compression test [3], and the fiber pull-out test [4]. They are carried out on microcomposite samples manufactured to this end and the interface strength is derived by means of simplified load transfer models, suitable for this type of microcomposite [5,2].
Although they were widely used in the past, it was always recognized that the local environment in these microcomposites was not representative of the mechanical and physico-chemical conditions of actual composites. For instance, the fiber packing density, the thermal residual stresses and the polymer morphology crosslinking density, crystallite morphology may be very different, leading to significant changes in the interface properties [6].

The characterization techniques used in fiber tows were initially developed for ceramic- and metal-matrix composites. They use standard composite materials and include the fiber push-out test [7-13], the slice compression test [14], and the fiber push-in test $[15,6,16-18]$. The application of the slice compression test to polymer-matrix composites is not obvious while the push-out needs a cumbersome preparation of a very thin membrane $(\approx 50 \mu \mathrm{m})$, leading to the fiber push-in test as the best alternative. Moreover, recording load and displacement during the push-in of an individual fiber on a composite cross-section has become a routine task with the help of a flat-punch nanoindentor. However, the interpretation of experimental results to determine the interface properties is not straightforward and requires a detailed micromechanical model. The standard approximations are based on the shear-lag model [5,2] or on two-dimensional (axisymmetric) representations of the fiber push-in test $[7,17]$ which cannot take into account important factors such as the local environment (fiber packing) [18], the anisotropic elastic properties of the carbon fibers and the influence of the processing residual stresses as well as of the fiber-matrix friction after decohesion on the interface properties. 
As a result, the experimental techniques presented above can be applied to rank the interface properties of identical composites containing fibers with different surface treatments, but they hardly provide absolute values for the interface properties. This statement is supported by the incredibly large scatter in the interface properties of identical composites when they are measured with different techniques and by the fact that there is no accepted standard to measure interface properties in composites $[1,19]$. This investigation was aimed at overcoming these limitations through a detailed simulation of the fiber push-in test applicable to structural composites, i.e. unidirectional carbon-fiber composites containing a large volume fraction of reinforcements. With the help of the numerical analyses, a methodology was established to determine the interface shear strength by means of push-in tests taking into account the influence of fiber packing, residual stresses, and friction on the test. The methodology was finally applied to a C/epoxy composite.

\section{Theoretical background of the fiber push-in test}

The push-in test is carried out by loading an individual fiber with a flat-punch nanoindenter until interface fracture takes place (Fig. 1a). The applied load, $P$ and the fiber displacement, $u$, are continuously monitored during the test and the corresponding $P-u$ curve is depicted in Fig. 1b. This curve presents an " $\mathrm{S}$ " shape where the initial region corresponds to the zone with imperfect contact between the punch and the fiber. It is followed by a linear zone (with stiffness $S_{0}$ ) due to the elastic deformation of fiber and matrix which ends with the beginning of interface failure [18]. The interface strength, $\tau_{c}^{S L}$, can be determined from the critical load at the onset of nonlinearity, $P_{c}$, by means of the standard shear-lag model $[5,2,6,17]$ according to

$\tau_{c}^{S L}=\frac{n P_{c}}{2 \pi r^{2}}$

where $r$ is the fiber radius and $n$ is a parameter which depends on the fiber and matrix elastic properties and on the local fiber volume fraction, i.e. the constraint imposed by the neighboring fibers. Following the shear-lag model, $n$ can be determined from the stiffness of the elastic region in the $P-u$ curve, $S_{0}$, as $[6,9,18]$

$n=\frac{S_{0}}{\pi r E_{1}^{f}}$

where $E_{1}^{f}$ stands for the longitudinal elastic fiber modulus. The interface strength - according to the shear lag model - is thus given by,
$\tau_{c}^{S L}=\frac{S_{0} P_{c}}{2 \pi^{2} r^{3} E_{1}^{f}}$

While this strategy is appealing for its simplicity, it has been shown that the constraining effect of the surrounding fibers is not accurately taken into account, leading to very large errors in the case of composites with very large fiber volume fraction [18]. In addition, the shear-lag model was derived for isotropic elastic fibers while $C$ fibers are extremely anisotropic. Finally, other effects, such as thermal residual stresses or interface friction are neglected without proper justification.

\section{Numerical simulation of the fiber push-in test}

\subsection{Geometrical model and discretization}

Following the previous considerations, it was decided to carry out a detailed 3D numerical simulation of the fiber push-in test to propose a methodology to characterize the interface strength based on this test. Obviously, the numerical simulations cannot explore all the local environments and it was necessary to restrict the analyses to one configuration which could be easily found and tested experimentally. The cross-section of a $C$ fiber composite (Fig. 2) shows that hexagonal packings formed by one fiber surrounded by its six nearest neighbors are often found in high performance composites containing a large fiber volume fraction ( $>60 \%)$. The local fiber volume fraction within these arrangements is very high (close to the theoretical limit of $91 \%$ ) and, for this reason, the spatial distribution of the fibers is regular, with very little deviation from the hexagonal symmetry (Fig. 2b).

The geometry of the numerical model used to analyze the fiber push-in test is shown in Fig. 3a. It includes the central fiber of diameter $2 r=5 \mu \mathrm{m}$ surrounded by six nearest neighbor fibers in an hexagonal packing. The seven fibers are embedded in the matrix material and this central region is surrounded by a ring of a homogeneous medium, which stands for the composite. The whole model could have been represented by a wedge of $30^{\circ}$ but it was decided to use $60^{\circ}$ to account for indenters with different tip geometries (Berkovich or cube-corner). The radius of the wedge is $32 r$ while the model length parallel to the fibers, $L$, is $150 r$. It was checked that these dimensions are large enough so that the load-displacement curve during fiber push-in is insensitive to the wedge dimensions. It should be noted that the central fiber is not in contact with the nearest neighbors (Fig. $3 \mathrm{~b}$ ). The minimum
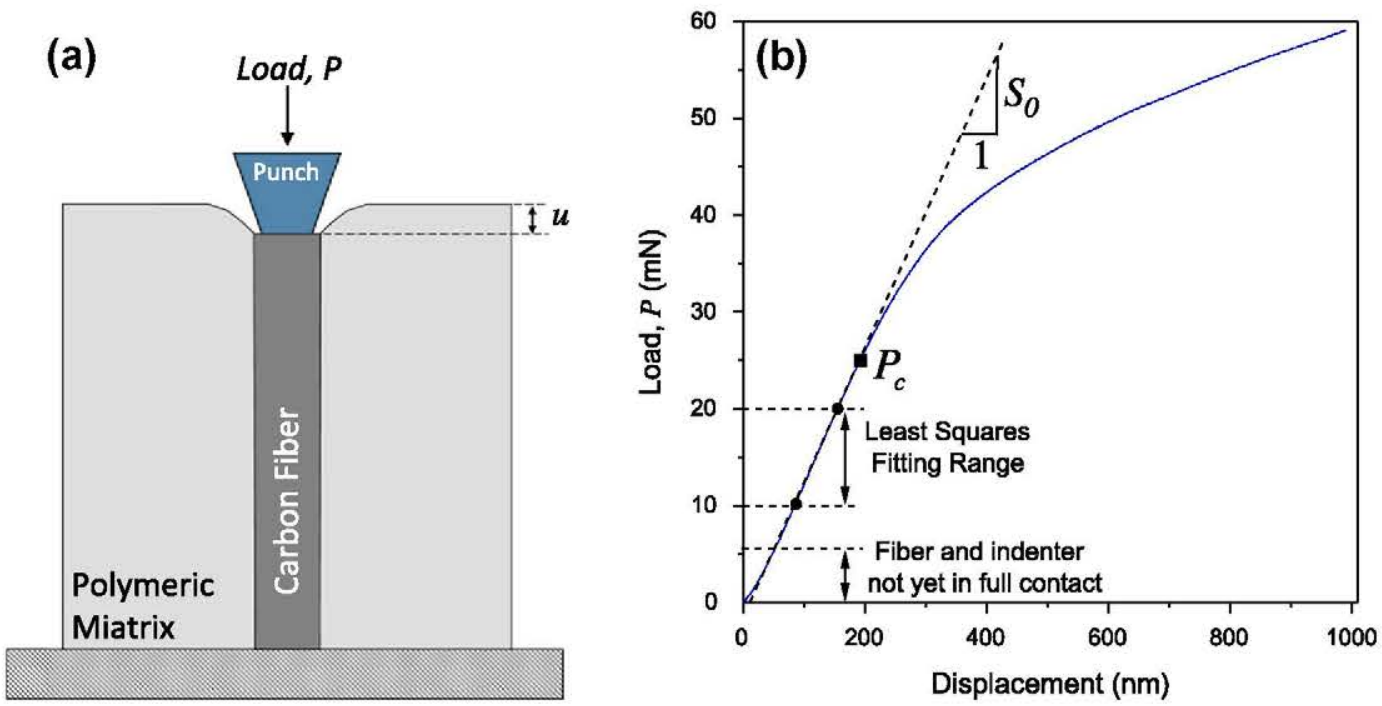

Fig. 1. (a) Schematic of the fiber push-in test. (b) Representative load-fiber displacement curve corresponding to a C/epoxy composite. 

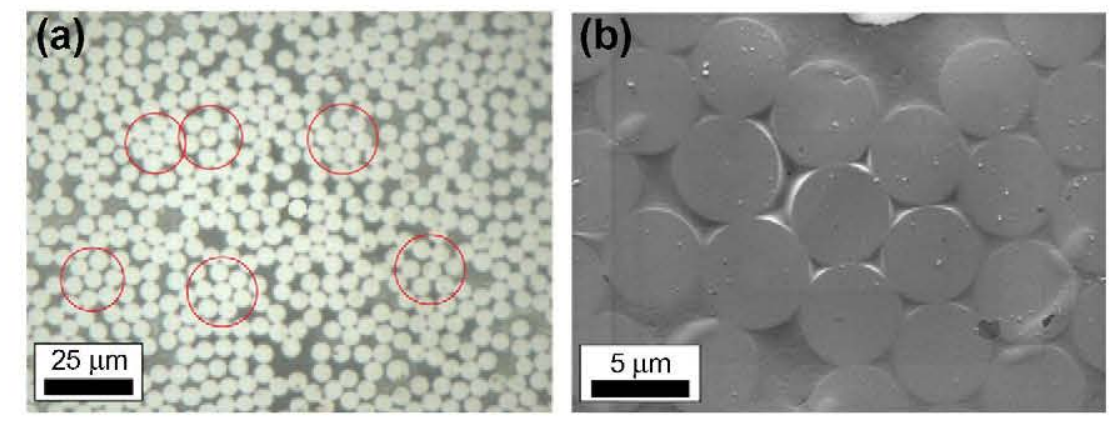

Fig. 2. (a) Optical micrograph of a cross-section of a carbon fiber/epoxy matrix composite. The regions enclosed by circles show hexagonal packings formed by one fiber surrounded by six nearest neighbors. (b) Scanning electron micrograph detail of one hexagonal packing showing the regular distribution of the neighbors around the central fiber.

\section{(a)}

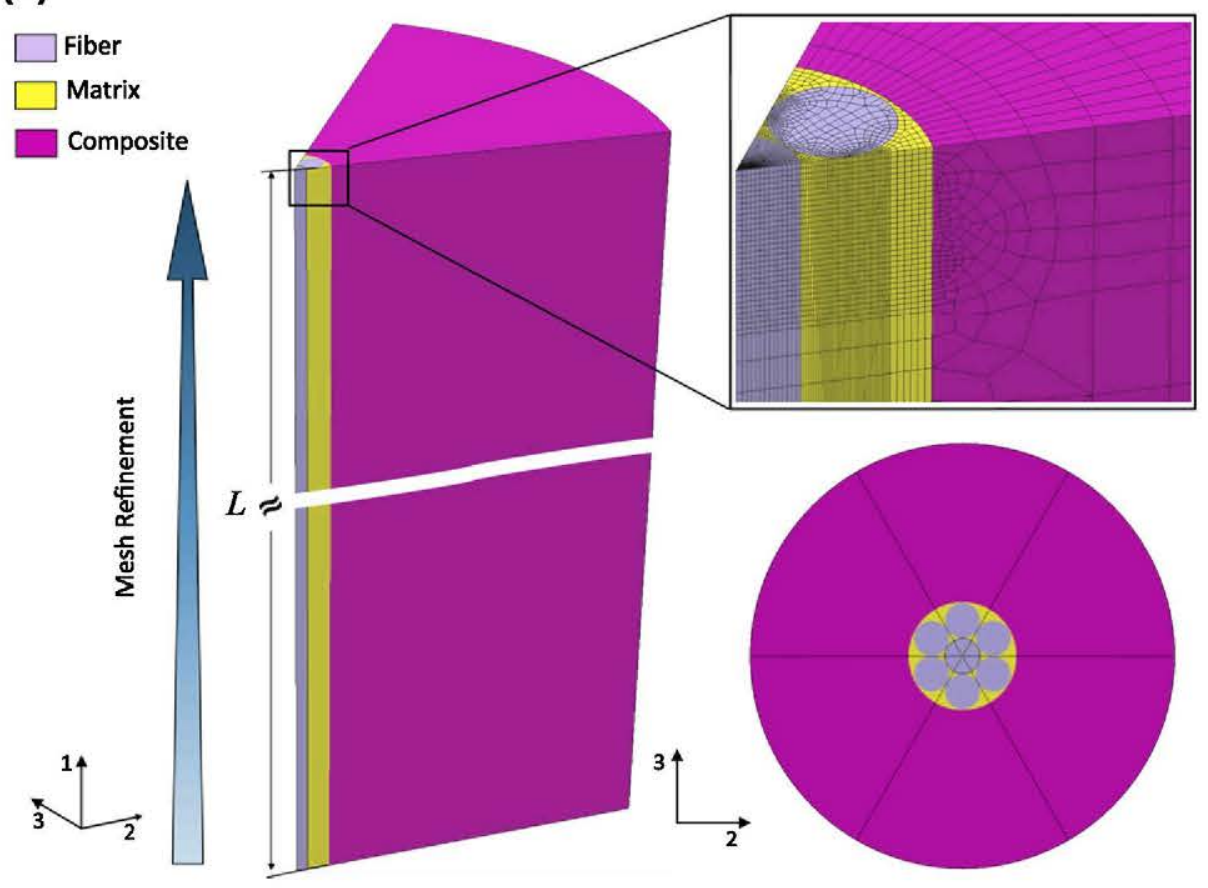

(b)
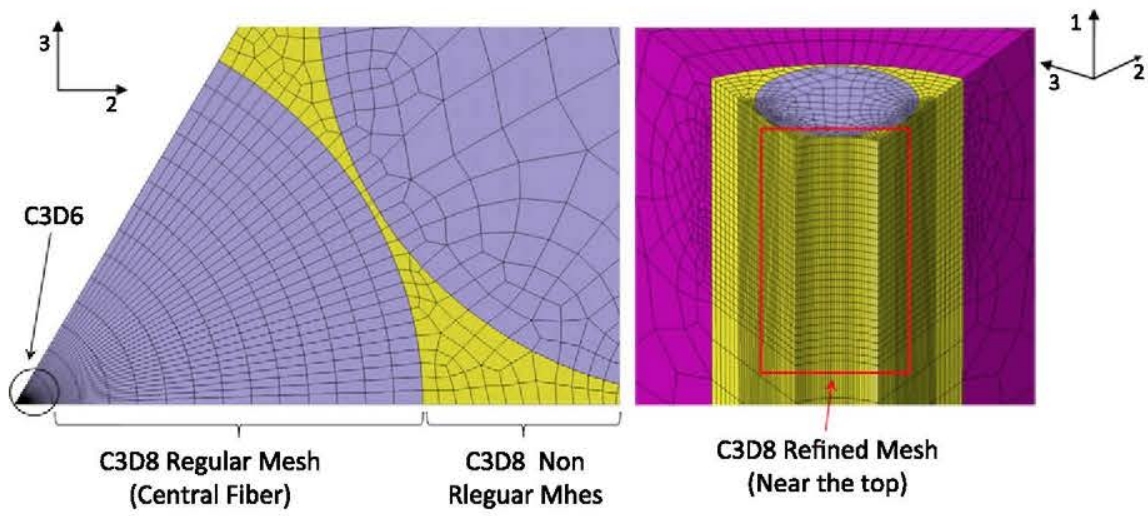

Fig. 3. (a) Geometry of the finite element model to simulate fiber push-in in composite cross-sections with an hexagonal fiber packing arrangement. (b) Detail of the finite element discretization around the central fiber.

thickness of the matrix layer in between is $0.02 r$, which was selected following the detailed observation of hexagonal fiber clusters in the scanning electron microscope and also attending to convergence problems during the simulations.
Matrix, fibers and the homogenized medium have been discretized with 8-node linear brick elements (C3D8) except for the axis of the central fiber, in which wedge elements (C3D6) have been used to ensure a structured mesh in the rest of the fiber (Fig. 3b). 
Full numerical integration has been used in both types of elements. Special care has been taken to obtain a very fine and homogeneous discretization throughout the model to resolve the shear stress gradients at the fiber/matrix interface during debonding, and the model is refined near the surface (Fig. 3b). In addition, cohesive surfaces are inserted between the central fiber and the matrix to account for interface decohesion and friction during the push-in test. The number of elements in the model is $\approx 73,000$. The density of the mesh as well as the aspect ratio of the elements were selected on the basis of an exhaustive analysis to ensure that the results were mesh-independent.

\subsection{Material properties}

The model includes three different solid materials (fibers, matrix and homogenized composite) and one fiber-matrix interface. Fibers behaved as thermo-elastic, transversally isotropic solids while the matrix was modeled as an isotropic, elastic solid. The corresponding thermo-elastic constants can be found in Table 1 . The matrix elastic constants, $E^{m}$ and $v^{m}$ and the fiber elastic modulus in the longitudinal direction, $E_{1}^{f}$, were provided by the composite manufacturer, while the thermal expansion coefficients were obtained from the literature [20]. The remaining elastic constants for the fiber were estimated using the Chamis model [21] from the elastic constants of unidirectional composite coupons assuming an average fiber volume fraction of $67 \%$. Plastic deformation of the matrix was not taken into account because detailed numerical analysis revealed that it did not influence the critical stress at the onset of fiber-matrix debonding for this particular hexagonal fiber arrangement, in which interface fracture was mainly controlled the high local fiber volume fraction. It should be noted, however, that matrix plasticity should be included to compute the interface shear strength in the case of isolated fibers or when the matrix shear flow stress is below $50 \%$ of the interface shear strength.

The interface behavior was characterized by a cohesive crack model, as in our previous analysis of interface fracture in composites [12,22,23]. The cohesive model relates the total stress acting on the interface, $t=\sqrt{\left\langle t_{n}\right\rangle^{2}+t_{t}^{2}+t_{s}^{2}}$, with the corresponding displacement jump, $\delta=\sqrt{\delta_{n}^{2}+\delta_{t}^{2}+\delta_{s}^{2}}$, where $t_{n}, t_{t}$ and $t_{s}$ are, respectively, the normal and tangential stresses transferred by the interface. 〈〉 stand for the Macaulay brackets, which return the argument if positive and zero otherwise, because compressive normal stresses do not open the crack. In addition, $\delta_{n}, \delta_{t}$ and $\delta_{s}$ are, respectively, the normal and tangential displacement jumps across the cohesive surface. The simplest constitutive equation for the cohesive crack is the bilinear model (Fig. 4). In the absence of damage, the interface behavior is linear with an initial stiffness, $K$, which is a numerical parameter large enough to ensure the displacement continuity at the interface and to avoid any modification of the stress fields in the absence of damage. The onset of damage is attained when the total stress acting on the interface reaches a critical value $t^{c}$ given by a quadratic criterion according to [24],

$\left\{\frac{\left\langle t_{n}\right\rangle}{t_{n}^{c}}\right\}^{2}+\left\{\frac{t_{t}}{t_{t}^{c}}\right\}^{2}+\left\{\frac{t_{s}}{t_{t}^{c}}\right\}^{2}=1$

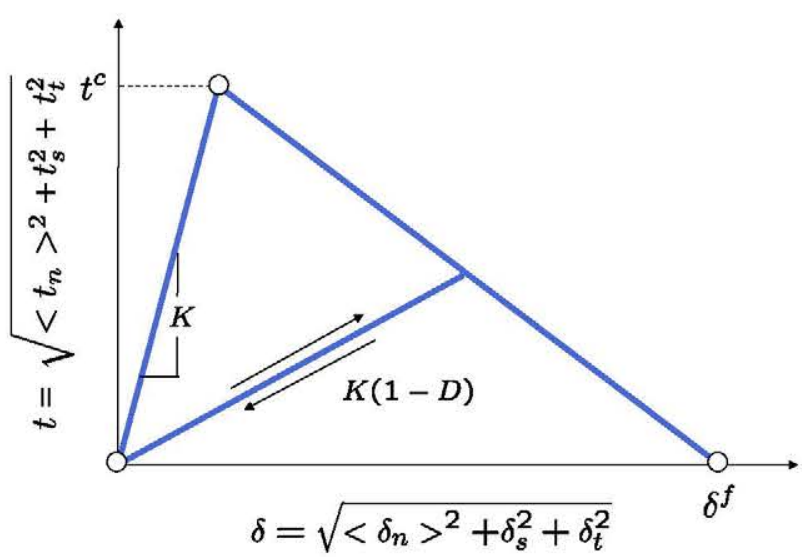

Fig. 4. Constitutive equation of the cohesive crack model for the interface.

where $t_{n}^{c}$ and $t_{t}^{c}$ stand for, respectively, the normal and shear interfacial strength. In the simulation of the fiber push-in test, normal stresses are always negligible as compared with shear stresses at the fracture point and it was found that the actual magnitude of $t_{n}^{c}$ did not modify the result of the simulations. So, for the sake of simplicity, the critical values of the normal and shear interfacial strength were set equal to each other, $t_{n}^{c}=t_{t}^{c}=\tau_{c}$, which will be taken as the actual interface strength throughout the paper.

Damage propagation leads to a progressive reduction in the stresses transferred through the interface as well as in the interface stiffness (Fig. 4). The actual reduction of the stress transferred by the cohesive crack is defined by the slope of the softening region in the constitutive equation, which depends on $t^{c}$ and the interface fracture toughness $\Gamma=0.5 t^{c} f^{f}$ (the area under the curve). It was assumed that the energy necessary to break the interface was independent of the loading path. Once the interface element was completely broken, the tangential sliding between matrix and fiber was opposed by Coulomb friction, characterized by the friction coefficient $\mu$.

\subsection{Analysis}

Simulations were carried out using Abaqus/Standard [25] within the framework of the finite deformations theory with the initial unstressed state as reference. The boundary conditions of the wedge (Fig. 3a) were the following: the vertical displacements of the bottom surface were constrained, while symmetry boundary conditions were applied on the vertical lateral surfaces, perpendicular to the circumferential direction. Finally, the vertical surface perpendicular to the radial direction as well as the upper surface of the wedge were stress free. Fiber indentation was simulated by means of the vertical displacement of a rigid, cylindrical flat punch of $3 \mu \mathrm{m}$ in diameter, similar to the one used in the experiments. Friction between the punch and the fiber was neglected. Additionally, the simulations revealed that the debonding process is independent of the flat punch radius when the ratio between the indenter and the fiber diameters was equal to or higher than 0.6 .

Table 1

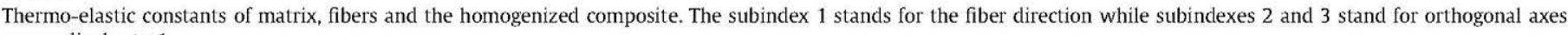
perpendicular to 1 .

\begin{tabular}{|c|c|c|c|c|c|c|c|}
\hline Material & $E_{1}(\mathrm{GPa})$ & $E_{2}(\mathrm{GPa})$ & $G_{12}(\mathrm{GPa})$ & $\mathrm{G}_{23}(\mathrm{GPa})$ & $v_{12}$ & $\alpha_{1}\left(10^{-6} /{ }^{\circ} \mathrm{C}\right)$ & $\alpha_{2}\left(10^{-6} /{ }^{\circ} \mathrm{C}\right)$ \\
\hline Fiber & 296 & 17.5 & 19 & 6 & 0.28 & -0.4 & 5.6 \\
\hline Matrix & 3 & & & & 0.35 & 40 & 40 \\
\hline Composite & 199.3 & 9.4 & 4.9 & 3.4 & 0.30 & 12.9 & 17.0 \\
\hline
\end{tabular}




\section{Results}

The 3D model presented above was used to assess the influence of different factors (shear interface strength and fracture energy, curing stresses, and friction) on the $P-u$ curve provided by the push-in test in order to establish a reliable methodology to obtain the interface properties from this test.

\subsection{Effect of the interface shear strength}

The first set of simulations was aimed at determining the point of the $P-u$ at which interface decohesion began. Curing stresses and friction were neglected and the interface toughness, $\Gamma$, was set to $20 \mathrm{~J} / \mathrm{m}^{2}$. Analyses with different values of the interface shear strength $50 \mathrm{MPa} \leqslant \tau_{c} \leqslant 100 \mathrm{MPa}$ were carried out and the corresponding $P-u$ curves are plotted in Fig. 5a. All of them present the same initial elastic stiffness, $S_{0}$, followed by a non-linear region which stands for the propagation of the interface crack from the upper surface into the bulk. All the curves converge to a plateau with constant load once the steady-state situation for crack prop- agation has been attained and crack growth is controlled by the interface toughness. The symbols in Fig. $5 \mathrm{a}$ indicate the critical load, $P_{c}$, corresponding to the onset of interface decohesion for the composites with different interface strength. $P_{C}$ was determined at the intersection of the $P-u$ curve with a straight line that passes from two points of the $P-u$ curve determined from two parallel lines with the initial stiffness $S_{0}$ drawn with offset displacements of $2 \%$ and $10 \%$ (Fig. 5b). This definition of the $P_{C}$ (somewhat arbitrary) provided very good estimations for the critical load at the onset of debonding in all the numerical simulations and can be readily applied to an experimental $P-u$ curve.

Following this criterion, the actual interface shear strength, $\tau_{c}$, is plotted in Fig. $5 \mathrm{c}$ as a function of the interface strength computed according to the shear lag model, $\tau_{c}^{S L}$, (Eq. (3)) from the values $P_{c}$ and $S_{0}$ in Fig. 5a. While $\tau_{c}^{\text {SL }}$ is readily obtained from the push-in test, it seriously underestimates the actual interface strength in the case of carbon fiber composite reinforced with a high volume fraction of fibers. It is worth noting, however, that $\tau_{c}$ varies linearly with $\tau_{S L}$ according to $\tau_{c}=1.92 \tau_{c}^{S L}$ in the range of interface strengths studied and this opens the possibility to obtain $\tau_{c}$ from $\tau_{c}^{S L}$. To this end, it is necessary to ascertain the influence of other factors (interface
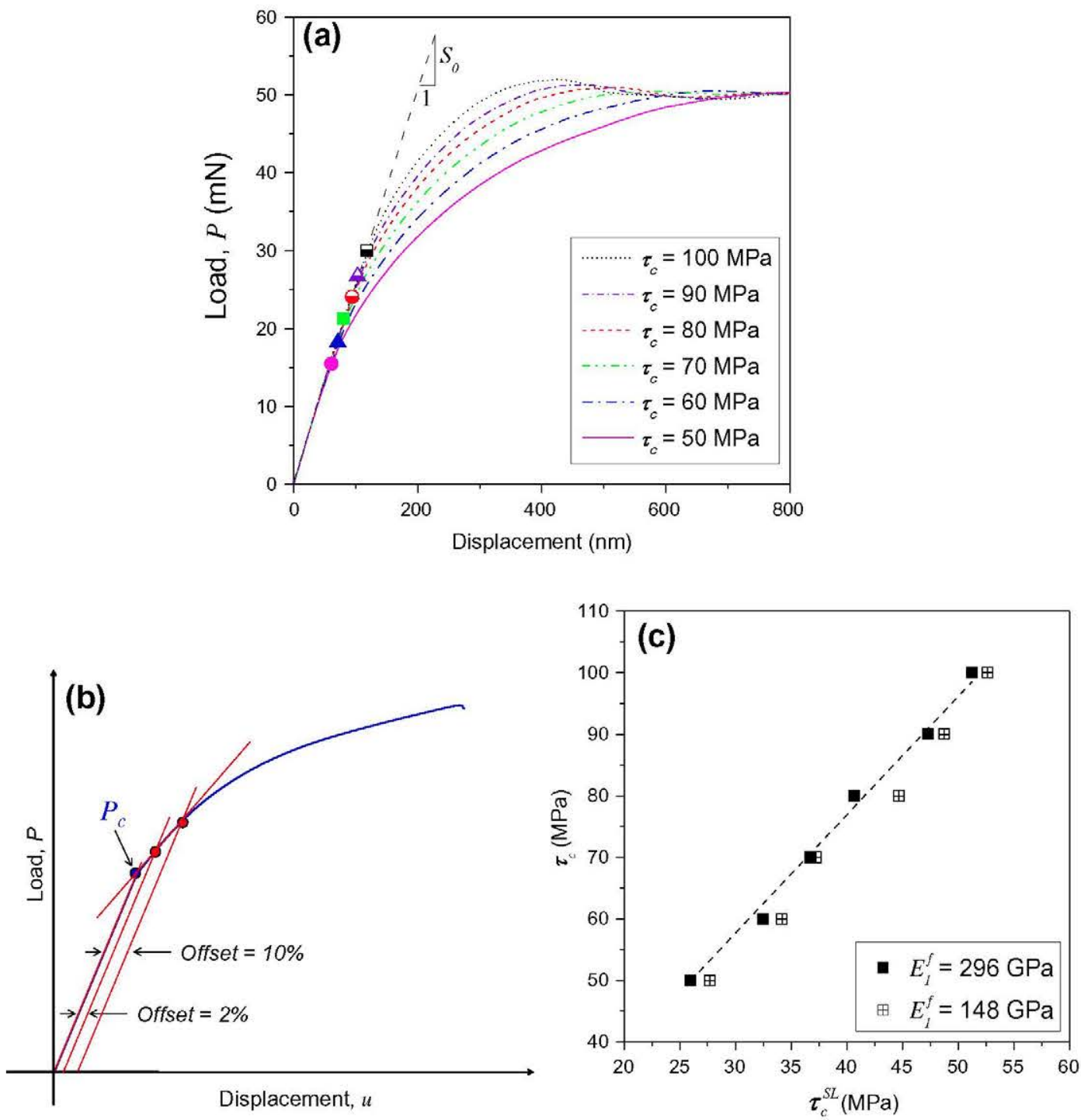

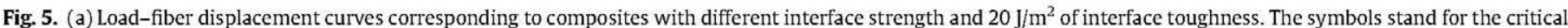

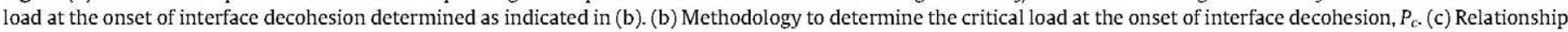
between the shear interface strength computed from the shear-lag model, $\tau_{c}^{S t}$ ) (Eq. (3)) and the actual shear interface strength, $\tau_{c}$. 
toughness and friction as well as curing stresses) on this relationship.
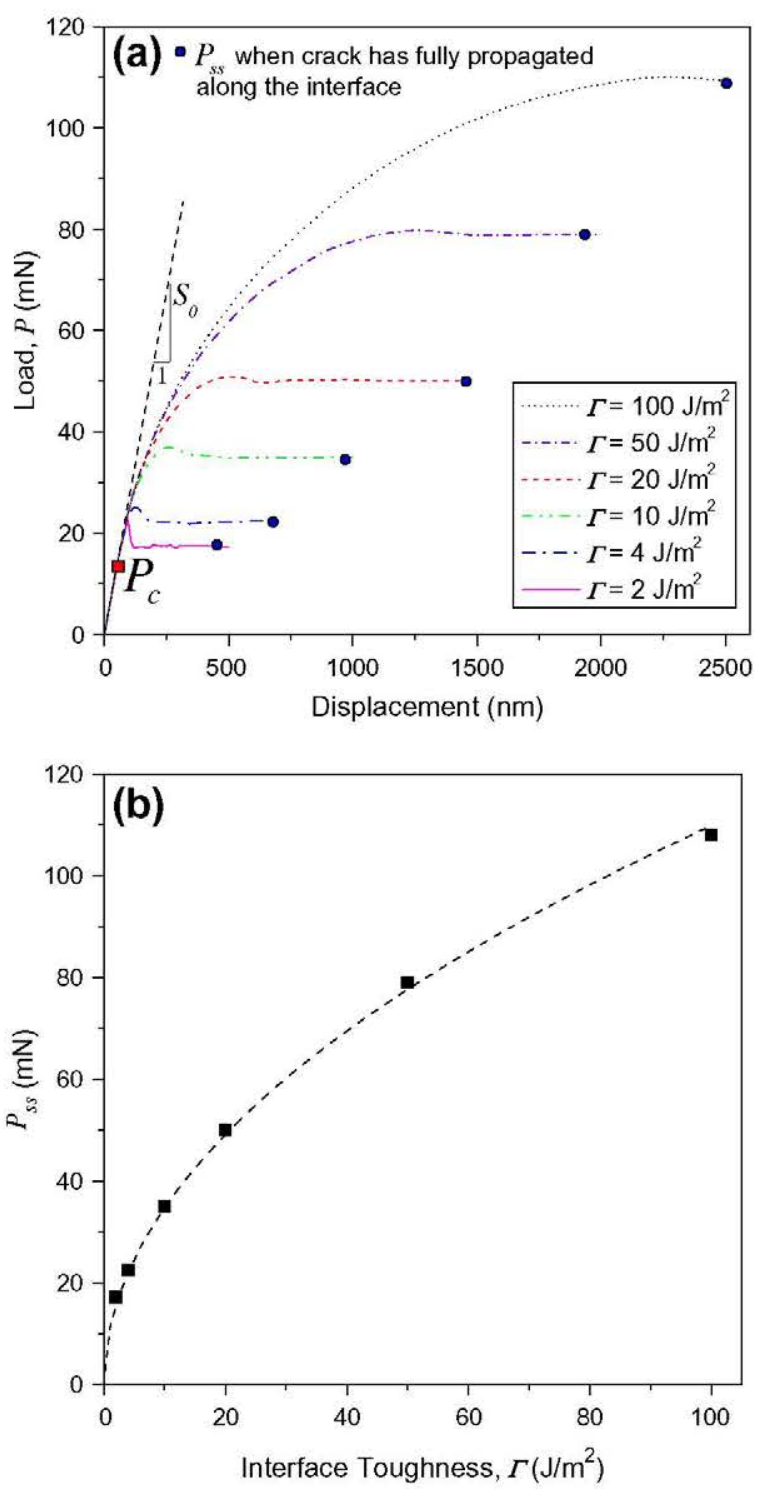

Fig. 6. (a) Load-fiber displacement curves corresponding to composites with different interface toughness and $80 \mathrm{MPa}$ of interface shear strength. The square symbol stands for the critical load at the onset of interface decohesion, $P_{c}$, computed as indicated in Fig. 5b. The circles at the end of the curve indicate the critical load for steady-state crack propagation, $P_{s s}$ ( (b) Influence of the interface toughness, $\Gamma$, on the critical load for steady-state crack propagation, $P_{s s}$. The broken line stands for a parabolic fitting according to the energy balance model of Jiang and Penn [26].
While the elastic modulus of epoxy resins is always of the order of a few GPa, the longitudinal elastic modulus of carbon fibers may vary significantly in the range $150 \mathrm{GPa}<E_{1}^{f}<350 \mathrm{GPa}$ and it was important to assess the influence of this parameter. To this end, a new set of simulations was carried out in which $E_{1}^{f}$ was reduced by half and the corresponding results are plotted in Fig. 5c. They show that the relationship between $\tau_{c}$ and $\tau_{c}^{S L}$ is not significantly influenced by the longitudinal modulus of the carbon fiber, whose effect is already included in the value of $\tau_{S L}$, Eq. (3).

\subsection{Effect of the interface toughness}

The $P-u$ curves computed for an interface with a critical shear strength $\tau=80 \mathrm{MPa}$ and different values of the interface toughness in the range $2 \mathrm{~J} / \mathrm{m}^{2} \leqslant \Gamma \leqslant 100 \mathrm{~J} / \mathrm{m}^{2}$ are plotted in Fig. 6a. Brittle interfaces $\left(\Gamma<10 \mathrm{~J} / \mathrm{m}^{2}\right)$ present a maximum in the $P$ - $u$ curve as the stress necessary to initiate the crack is higher than the plateau load under steady-state crack propagation. The non-linear region of the $P-u$ curve and the plateau load, $P_{s s}$ increase rapidly with $\Gamma$, and it is worth noting that $P_{S S}$ is proportional to $\sqrt{\Gamma}$, in agreement with the energy balance model of Jiang and Penn [26] (Fig. 6b). One important result of these simulations is that the critical load at the onset of decohesion, $P_{c}$, as defined above, is independent of the interface toughness and the ratio $\tau_{c} / \tau_{c}^{S L}$ in Fig. $5 c$ is valid regardless of the interface toughness.

\subsection{Effect of curing stresses and friction}

Thermal residual stresses develop in composites upon cooling after curing as a result of the thermal expansion mismatch between matrix and fibers. Carbon fibers expand slightly in the longitudinal direction upon cooling while the matrix shrinks (Fig. 7), and this leads to a build-up of shear stresses along the interface which has to be overcome during indentation to promote interface decohesion. In addition, the fiber is clamped within the matrix by compressive stresses normal to the interface, which can increase friction after decohesion. Both factors are expected to increase the indentation load necessary to initiate and propagate the interface crack and they are analyzed below.

The thermal residual stresses were introduced in the model by means of an initial elastic thermal step, in which the temperature was reduced from $180^{\circ} \mathrm{C}$ to $20^{\circ} \mathrm{C}$. Indentation of the fiber was simulated afterwards and the $P-u$ curves with and without curing stresses are plotted in Fig. 8a for a composite with an interface with $\tau_{c}=80 \mathrm{MPa}, \Gamma=20 \mathrm{~J} / \mathrm{m}^{2}$ and $\mu=0$. While the residual stresses do not modify the initial stiffness, they increase the critical load for the onset of decohesion as well as the plateau load for steady-state crack propagation. As shown in Fig. 8c, the influence of the curing stresses on the interface shear strength is linear and can be accounted for according to

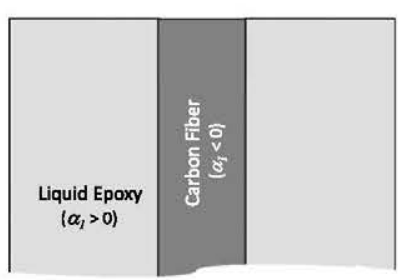

Before Curing $\left(180^{\circ} \mathrm{C}\right)$

(a)

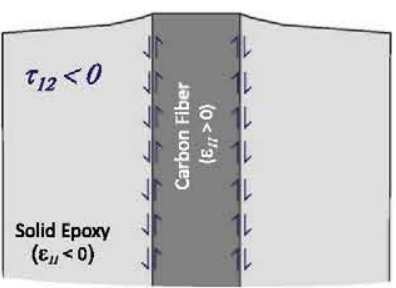

After Curing and Cooling

(b)

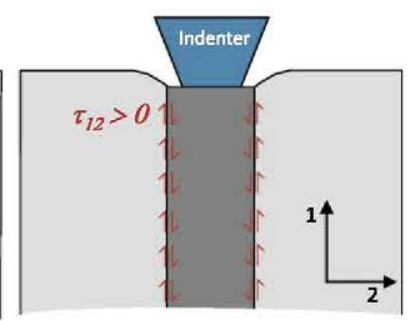

During Push-In Test

(c)

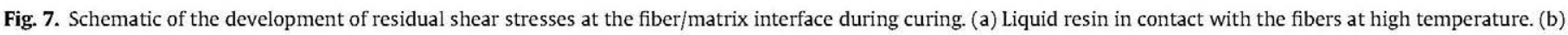
Interface shear stresses at ambient temperature after curing and cooling. (c) Interface shear stresses generated during the push-in test. 

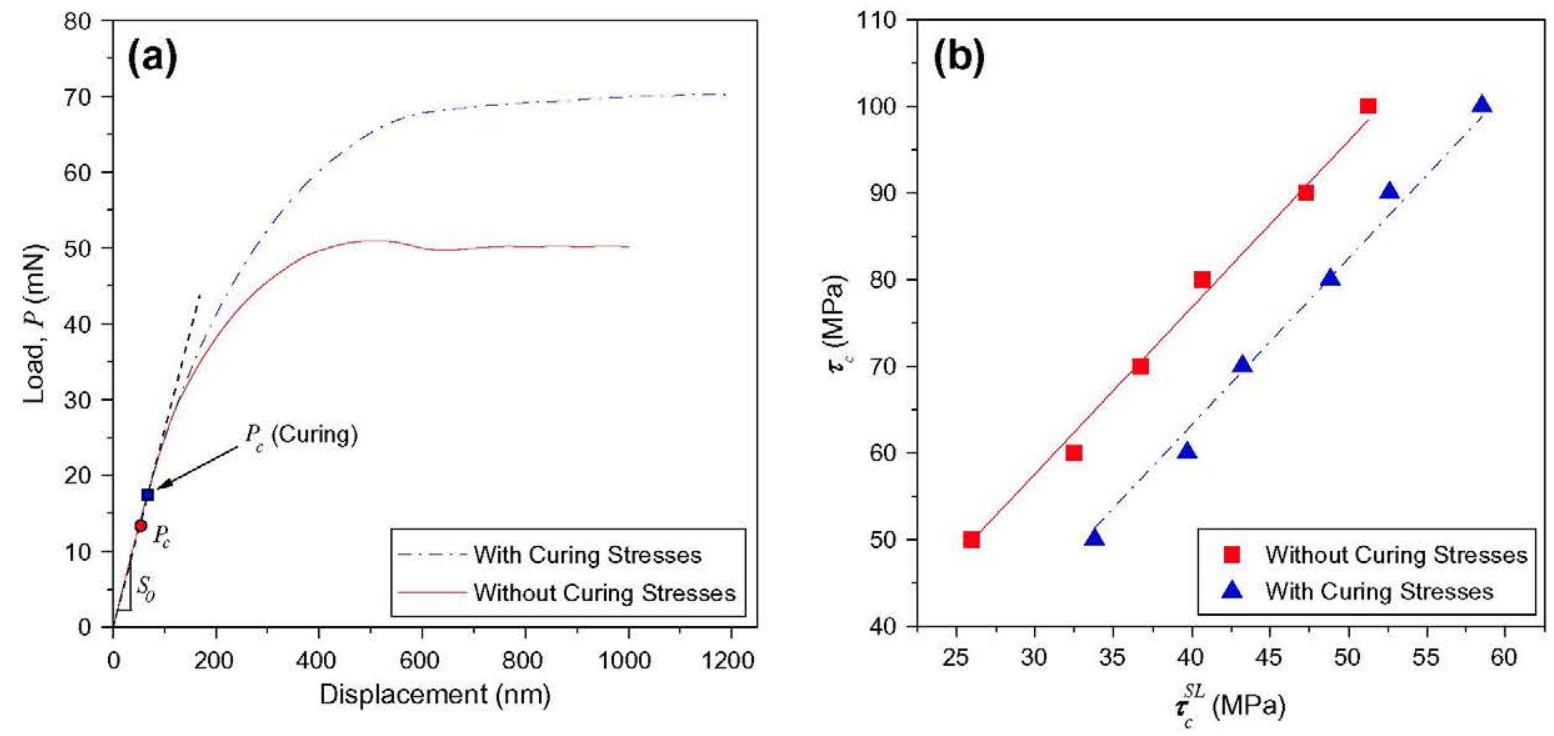

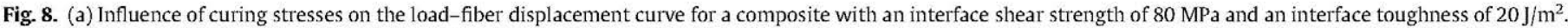

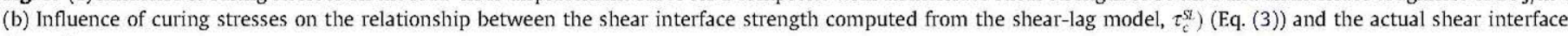
strength, $\tau_{c}$.

$\tau_{c}=1.92 \tau_{c}^{S L}-0.085 \Delta T$

where $\Delta T$ stands for the temperature change in ${ }^{\circ} \mathrm{C}$ and $\tau_{c}$ and $\tau_{c}^{S L}$ are expressed in MPa.

Obviously, the curing stresses clamp the fibers and also enhance the energy dissipation by friction after complete interface debonding. The influence of these stresses on the $P-u$ curve can be found in Fig. 9 for a composite with a shear interface strength and a fracture toughness of $80 \mathrm{MPa}$ and $20 \mathrm{~J} / \mathrm{m}^{2}$, respectively. The shear friction is assumed to depend on the normal stress at the interface, $\sigma_{r}$, according to

$\tau_{f r}=\mu \sigma_{r}$

for different values of the friction coefficient in the range $0 \leqslant \mu \leqslant 1$. Interface friction increases the load necessary to propagate the crack and the plateau corresponding to the self-similar propagation of the crack under steady-state conditions is never achieved be-

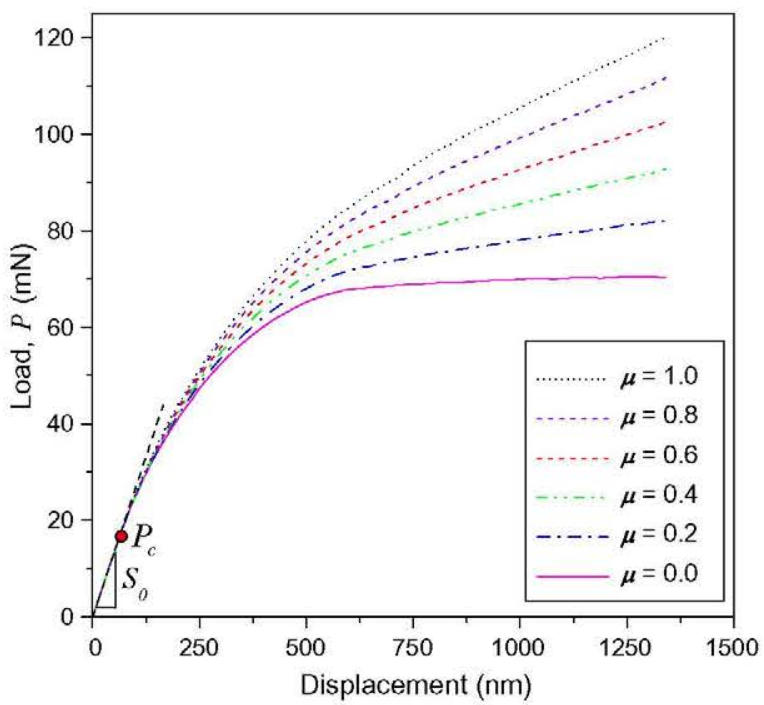

Fig. 9. Influence of interface friction on the load-fiber displacement curve for a composite with an interface shear strength of $80 \mathrm{MPa}$ and an interface toughness of $20 \mathrm{~J} / \mathrm{m}^{2}$ cause the larger the debonded zone, the higher the friction contribution. Fortunately, this effect is only important once the interface has been broken and the critical load at the onset of decohesion was not affected by the friction coefficient.

\section{Methodology to measure the interface strength by means of the fiber push-in test}

The numerical simulations presented above show that the shear interface strength can be determined from fiber push-in tests in composite materials with high accuracy under certain conditions. They are summarized below, together with the test methodology:

- This methodology to obtain the fiber/matrix interface strength is applicable to unidirectional fiber-reinforced composites. Push-in tests on individual fibers have to be carried out in fibers clusters with hexagonal symmetry and a very high fiber volume fraction to ensure reproducibility and also to apply the results of the numerical simulations. These regions can be easily found within fiber tows.

- The critical load at the onset of fiber/matrix debonding, $P_{c}$, is determined from the load-fiber displacement curve using the construction depicted in Fig. 5b. The numerical simulations have demonstrated that this value of $P_{c}$ coincides with the beginning of decohesion for a wide range of interface stresses. In addition, the loading stiffness, $S_{0}$, can be determined from the slope of the linear region of the $P-u$ curve as indicated in Fig. 1b.

- The interface strength corresponding to the classical shear lag model is obtained from Eq. (3) from $P_{c}, S_{0}$, the fiber radius, $r$, and the longitudinal fiber modulus, $E_{1}^{f}$. The actual interface strength, $\tau_{c}$, is obtained from this value using Eq. (5) to account for the anisotropy of the fibers and the curing stresses.

\subsection{Application}

This methodology has been used to determine the interface strength in an unidirectional T800 carbon fiber/epoxy composite. Pre-impregnated sheets of $300 \times 300 \mathrm{~mm}^{2}$ were purchased from Hexcel and composite laminates $[0]_{4}$ were consolidated in 


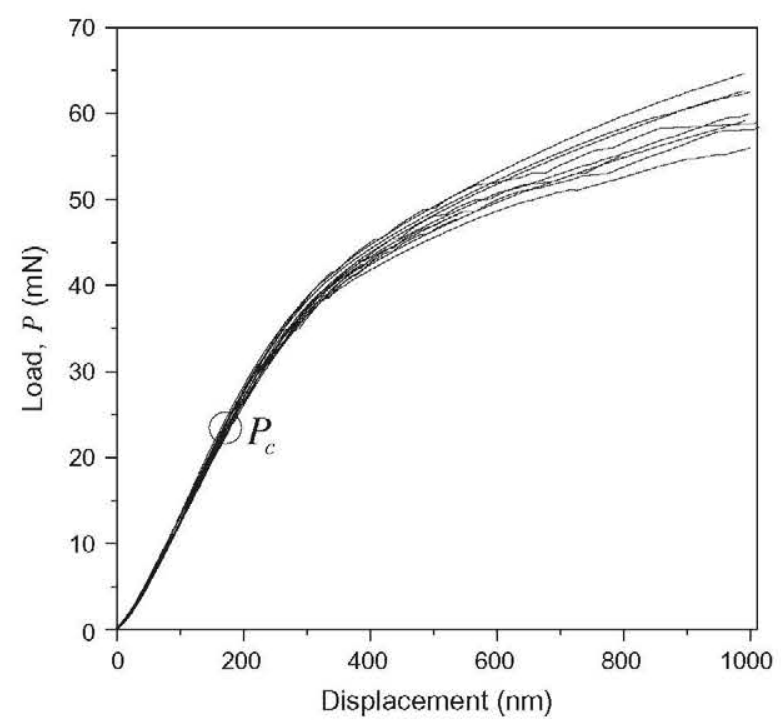

Fig. 10. Experimental load-fiber displacement curve for a C/epoxy composite.

autoclave at $180^{\circ} \mathrm{C}$ and 7 bars for $120 \mathrm{~min}$. They were cooled at $2{ }^{\circ} \mathrm{C} / \mathrm{min}$ releasing the applied pressure at the end of the cycle. The total thickness of the laminate was $1 \mathrm{~mm}$ and the nominal fiber volume fraction was $67 \%$. Cross-sections perpendicular to the fibers were polished on SiC paper to 1000 grit finish followed by a diamond slurry up to $1 \mu \mathrm{m}$.

Fifteen push-in tests were carried out on individual fibers which were arranged in an hexagonal pattern (Fig. 2). Indentations were performed with a Hysitron T1950 nanoindenter equipped with a flat punch of $3 \mu \mathrm{m}$ in diameter. Indentations were carried out at a constant displacement rate of $10 \mathrm{~nm} / \mathrm{s}$ up to a maximum depth in the range $400 \mathrm{~nm}$ to $1 \mu \mathrm{m}$. The corresponding $P-u$ curves are shown in Fig. 10: they show very good reproducibility, particularly in the linear region and at the onset of non-linearity, indicating that the indentation of hexagonal fiber clusters is robust.

The initial stiffness $S_{0}=148.5 \pm 4.5 \mu \mathrm{N} / \mathrm{nm}$ was determined from the linear region of the $P-u$ curve as indicated in Fig. 1b and the critical load for debonding, $P_{C}=24.4 \pm 1.7 \mathrm{mN}$, from the construction depicted in Fig. 5b. The experimental scatter was in both cases below $10 \%$, which can be considered very accurate for this type of tests. The critical shear strength according to the shear lag model, $\tau_{c}^{S L}$, was determined from these values from Eq. (3). The actual interface shear strength, $\tau_{c}$, taking into account the anisotropy of the carbon fibers and the influence of the curing stresses, was obtained from Eq. (5), leading to a value of $63 \pm 5 \mathrm{MPa}$, which is reasonable for these types of composites and in good agreement with the results in the literature in similar C/epoxy composites [27,28]. In contrast, other investigations [29] reported very low values (30-35 MPa) for the interface strength of similar C/epoxy composites. Their data was obtained from push-in tests by applying the shear-lag model and very probably underestimated the interface strength because neither the elastic fiber anisotropy nor the internal stresses were accounted for.

\section{Concluding remarks}

Three-dimensional finite element simulations were carried out to ascertain the influence of interface properties (strength, toughness and friction), fiber elastic anisotropy and curing stresses on the fiber push-in test. Numerical analyses were restricted to a particular fiber configuration made up of a close-packed hexagonal fiber arrangement. This configuration is often found in cross- sections of unidirectional fiber-reinforced composites. It was found that the critical load at the onset of decohesion, $P_{c}$ depended on the interface strength and the curing stresses but was independent of the interface toughness and friction coefficient. The interface strength derived from the $P_{c}$ using the classical shear lag model underestimated (by a factor of $\approx 2$ ) the actual interface strength of the material because it did not take into account the influence of the fibers' elastic anisotropy and of the curing stresses. Based on the results of the numerical simulations, a simple correction of the shear model was derived and a new methodology was proposed to obtain the fiber/matrix interface shear strength and it was successfully applied to a high volume fraction C/epoxy composite.

The main advantages of the new methodology are that it can be easily performed in standard composite specimens and can provide quantitative values of interface shear strength, as opposed to qualitative estimations. Quantitative values are necessary to establish absolute comparisons between interfaces in different materials and are also very useful to feed multiscale modeling tools which attempt to predict the mechanical behavior of composite plies, laminates and components from the properties and spatial arrangement of fiber, matrix and interfaces in the composite [30].

The numerical simulations also pointed out the limitations of the push-in test to characterize the interface properties. Firstly, it does not provide information about the normal interface strength, which seems to be critical for the composite properties under transverse tension [27,31]. Secondly, it will not be easy to develop a parallel methodology to extract information about the interface toughness and friction coefficient because of the strong coupling between them and also because friction is very dependent on the curing stresses, which are not always easy to estimate. Thus, further developments are required in this area and, in particular, recent advances in mechanical testing of micron-sized specimens mechanized from the actual composite samples using focus ion beam techniques are very promising to characterize interface properties.

\section{Acknowledgements}

This investigation was supported by the Ministerio de Ciencia e Innovación de España through the Grant MAT 2009-14396, by the Comunidad de Madrid through the program ESTRUMAT (S2009/ MAT-1585), and by the European Community's Seventh Framework Programme FP7/2007-2013 under grant agreement 213371 (MAAXIMUS, http://www.maaximus.eu). M.R. acknowledges the support from the Comunidad de Madrid for the fellowship to carry out his doctoral thesis.

\section{References}

[1] Kim J-K, Mai YW. Engineered interfaces in fiber reinforced composites. Elsevier; 1998.

[2] Kelly A, Tyson WR. Tensile properties of fibre-reinforced metals: copper/ tungsten and copper/molybdenum. J Mech Phys Solids 1965;13:329-38.

[3] Broutmari LJ. Measurement of the fiber-polymer matrix interfacial strength. Interfaces in composites, vol. 452. ASTM International; 1969. p. 27.

[4] Miller B, Muri P, Rebenfeld L. A microbond method for determination of the shear strength of a fiber/resin interface. Compos Sci Technol 1987;28:17-32.

[5] Cox HL. The elasticity and strength of paper and other fibrous materials. Brit J Appl Phys 1952;3:72-9.

[6] Kharrat M, Chateauminois A, Carpentier L, Kapsa P. On the interfacial behaviour of a glass/epoxy composite during a micro-indentation test: assessment of interfacial shear strength using reduced indentation curves. Compos A: Appl Sci Manuf 1997;28:39-46.

[7] Kerans RJ, Parthasarathy TA. Theoretical analysis of the fiber pullout and pushout tests. J Am Ceram Soc 1991;74:1585-96.

[8] Mackin TJ, Yang J, Warren PD. Influence of fiber roughness on the sliding behavior of sapphire fibers in TiAl and glass matrices. J Am Ceram Soc $1992 ; 75: 3358-62$.

[9] Bechel VT, Sottos NR. Application of debond length measurements to examine the mechanics of fiber pushout. J Mech Phys Solids 1998;46:1675-97. 
[10] Chandra N, Ghonem H. Interfacial mechanics of push-out tests: theory and experiments. Compos A: Appl Sci Manuf 2001;32:575-84.

[11] González C, LLorca J. Micromechanical modelling of deformation and failure in Ti-6Al-4V/SiC composites. Acta Mater 2001;49:3505-19.

[12] González C, Llorca J. Numerical simulation of the fracture behavior of $\mathrm{Ti} /$ Siccomposites between $20^{\circ} \mathrm{C}$ and $400^{\circ} \mathrm{C}$. Metall Mater Trans A 2007;38:169-79.

[13] Rollin M, Jouannigot $S$, Lamon J, Pailler R. Characterization of fibre/matrix interfaces in carbon/carbon composites. Compos Sci Technol 2009;69:1442-6.

[14] Hsueh C, Brandon D, Shafry N. Experimental and theoretical aspects of slice compression tests. Mater Sci Eng A 1996;205:91-100.

[15] Mandell JF, Chen JH, McGarry FJ. A microdebonding test for in situ assessment of fibre/matrix bond strength in composite materials. Int J Adhes Adhes $1980 ; 1: 40-4$

[16] Nishikawa M, Okabe T, Hemmi K, Takeda N. Micromechanical modeling of the microbond test to quantify the interfacial properties of fiber-reinforced composites. Int J Solids Struct 2008;45:4098-113.

[17] Zidi M, Carpentier L, Chateauminois A, Sidorof F. Quantitative analysis of the micro-indentation behaviour of fibre-reinforced composites: development and validation of an analytical model. Compos Sci Technol 2000;60:429-37.

[18] Molina-Aldareguía JM, Rodríguez M, González C, LLorca J. An experimental and numerical study of the influence of local effects on the application of the fibre push-in test. Philos Mag 2011;91:1293-307.

[19] Zhou X-F, Wagner HD, Nutt SR. Interfacial properties of polymer composites measured by push-out and fragmentation tests. Composites Part A 2001;32:1543-51.

[20] Andrews EW, Garnich MR. Stresses around fiber ends at free and embedded ply edges. Compos Sci Technol 2008;68:3352-7.
[21] Chamis CC. Mechanics of composite materials: past, present, and future. NASA Technical Memorandum 100793.

[22] Totry E, González C, LLorca J. Failure locus of fiber-reinforced composites under transverse compression and out-of-plane shear. Compos Sci Technol 2008;68:829-39.

[23] Totry E, Molina-Aldareguía JM, González C, LLorca J. Effect of fiber, matrix and interface properties on the in-plane shear deformation of carbon-fiber reinforced composites. Compos Sci Technol 2010;70:970-80.

[24] Ogihara S, Koyanagi ]. Investigation of combined stress state failure criterion for glass fiber/epoxy interface by the cruciform specimen method. Compos Sci Technol 2010;70:143-50.

[25] Abaqus. Analysis user's manual, version 6.10, Simulia; 2011.

[26] Jiang KR, Penn LS. Improved analysis and experimental evaluation of the single filament pull-out test. Compos Sci Technol 1992;45:89-103.

27] Hobbiebrunken $\mathrm{T}$, hojo M, Adachi T, De Jong C, Fiedler B. Evaluation of interfacial strength in CF/epoxies using FEM and in-situ experiments. Composites Part A 2006;37:2248-56.

28] Herrera-Franco PJ, Drzal LT. Comparison of methods for the measurement of fibre/matrix adhesion in composites. Composites 1992;23:2-27.

[29] Haeberle DC, Lesko JJ, Case SW, Riffle JS, Verghese KE. The use of a modified microindentation technique to evaluate enviro-mechanical changes in composite interphase properties. J Adhes Sci Technol 2007;21:35-50.

[30] LLorca J, González C, Molina-Aldareguía JM, Segurado J, Seltzer R, Sket F, et al. Multiscale modeling of composite materials: a roadmap towards virtual testing. Adv Mater 2011;23:5130-47.

[31] Canal LP, González C, Segurado J, LLorca J. Intraply fracture of fiber-reinforced composites: microscopic mechanisms and modeling. Compos Sci Technol $2012 ; 72: 1223-32$ 